\title{
RELACIÓN ENTRE LA CARIES Y LOS PRINCIPALES MALESTARES EXPRESADOS POR ESCOLARES DE 6 AÑOS, SAN SEBASTIÁN-CUENCA 2016
}

\section{RELATIONSHIP BETWEEN CARIES AND ITS IMPACT ON DAILY PERFORMANCE IN SCHOOLS AGED 6 YEARS, SAN SEBASTIÁN-CUENCA 2016}

\author{
Gutierrez-Pulla Karen $^{1 *}$, Encalada-Verdugo Liliana ${ }^{2}$ \\ ${ }^{1}$ Odontóloga Rural en el Ministerio de Salud Pública de Ecuador. Ecuador \\ ${ }^{2}$ Doctora en Odontología, Especialista en docencia Universitaria, Magíster en Estomatología, Docente en la Universidad \\ Católica de Cuenca. Ecuador \\ *kegutierrezp71@est.ucacue.edu.ec
}

\begin{abstract}
Resumen
OBJETIVO: El objetivo de este estudio fue relacionar la caries y su impacto en los desempeños diarios en escolares de 6 años de la Parroquia San Sebastián del cantón Cuenca en el año 2016. MATERIALES Y MÉTODOS: La metodología aplicada fue de tipo descriptiva, transversal de campo y observacional. El investigador recibió una calibración previa, se aplicó el índice ceod y una parte del instrumento CHILD-OIDP para evaluar las principales molestias expresadas por los escolares. RESULTADOS: La muestra de 197 escolares pertenecientes a 11 escuelas de la Parroquia San Sebastián fue distribuida por sexos, siendo el $45 \%$ de sexo femenino y el $55 \%$ de sexo masculino. El promedio total del índice ceod fue de 5,25 +3,60 (alto), las mujeres presentaron un promedio de ceod de 5,08 $(+3,33)$ y los hombres de 5,39 (+3,82). Tras aplicar la condición de caries del instrumento CHILD-OIDP se encontró que el $61 \%$ de mujeres y el $52 \%$ de hombres sufrieron impactos en sus desempeños diarios. Los desempeños con mayor severidad de impacto fueron el comer con un 20,4\% y el lavarse los dientes con un $17,1 \%$, los niños presentaron al menos un desempeño diario impactado. CONCLUSIÓN: Tras relacionar la caries y su impacto en los desempeños diarios se demostró, que los escolares afectados por caries tienen 1.72 veces más probabilidades de presentar impactos en sus desempeños diarios y por lo tanto mayor afección en su calidad de vida que aquellos que no presentaron esta enfermedad.
\end{abstract}

Palabras clave: Caries, impacto, calidad de vida.

\begin{abstract}
AIM: The aim of this study was to relate cavities and their impact on daily performance in schoolchildren about 6 years old from the San Sebastian parish of the Cuenca canton in 2016. MATERIALS AND METHODS: The applied methodology was descriptive, transverse of field and observational type. The researcher received a previous calibration, It was applied the indexes of dmft and CHILD-OIDP by registering the data in the epidemiological profile of the odontology career. RESULTS: The sample of 197 students from 11 schools in San Sebastian parish was distributed by sexes, with 45\% female and 55\% male. The total average of dmft index was 5,25 +3,60 (high), girls presented an average dmft 5,08 $(+3,33)$ and boys 5,39 (+3,82). After applying the decay condition CHILD-OIDP instrument, it was discovered that $61 \%$ of girls and $52 \%$ of boys had suffered impacts on their daily performance. The performances with more severely impact were eat $20,4 \%$ and brushing teeth $17,1 \%$, so the kids got to know at least one impacted daily performance. CONCLUSIONS: After linking caries and their impact on daily performances, this showed that schoolchildren affected by caries have 1,72 times more probabilities presenting impact on their daily performance and greater affection in their quality of life than those who did not present this sickness.
\end{abstract}

Key words: Caries, impact, quality of life. 


\section{INTRODUCCIÓN}

La caries dental es un proceso patológico de origen multifactorial, infeccioso, transmisible que afecta a los tejidos duros del diente y que se caracteriza por un desequilibrio bioquímico debido a la acción de los microorganismos que integran la placa dental, el huésped y la cavidad bucal; que de no ser revertido, conduce a una cavitación y alteraciones del complejo dentino-pulpar.

Esta condición bucal se ha convertido en una de las enfermedades más crónicas a nivel mundial y nuestro país al igual que otros, se encuentra afectado por esta patología, así lo evidencian los datos obtenidos por parte de la entidad de Salud Pública, colocándola como la principal enfermedad bucal del Ecuador con mayor prevalencia en la infancia. ${ }^{1}$

Es así como la caries dental en la primera infancia produce consecuencias graves en la salud del infante como procesos infecciosos que causan dolor intenso, en algunos casos se requiere de atención urgente y hospitalización, lo que conlleva a una menor calidad de vida del niño debido a su baja salud oral. ${ }^{2}$

A su vez, de acuerdo a estudios realizados en niños que padecen de caries en su niñez, se pudo evidenciar que esta afecta rápidamente a todos los dientes temporales, causando la pérdida prematura de los mismos y generando una mayor probabilidad de que el infante la padezca nuevamente en la dentición permanente. . $^{3,4}$

Es así como la primera infancia se ha identificado como el periodo más importante del ser humano por sus efectos a lo largo de la vida. Loreto et $\mathrm{al}^{5}$ mencionan que los tres primeros años son fundamentales para la vida futura de un niño, para su salud, crecimiento y desarrollo.

Pero para que le niño presente condiciones de bienestar desde su infancia se requiere de la intervención de sus padres. ${ }^{6}$ Es aquí donde su enseñanza se ve reflejada, pues si el infante observa que sus cuidadores presentan hábitos alimenticios y de higiene, el niño aprendería de esto y por lo tanto establecería en él hábitos desde edades tempranas que beneficiarían tanto en su salud general como en su salud bucal. ${ }^{7}$ Es por ello que en los niños la autopercepción se establece mediante lo que el infante mira y escucha de sus padres. $^{8}$

Se entiende por autopercepción, la visión que una persona tiene de sí misma cuando se trata de evaluar las propias fuerzas, autoestima y lo que percibe de su medio en forma de aceptación o de rechazo. ${ }^{9}$

La autopercepción y el autoestima en el infante cosiste en la sensación de bienestar consigo mismo y con su cuerpo, estar conforme con su género, mantener un estado emocional equilibrado, ser partícipe de su familia, tener la seguridad de que puede cumplir con sus actividades y que todos sus requerimientos siempre serán atendidos. ${ }^{10}$

Las investigaciones en el campo de la psicología afirman que los 6 primeros años de edad son vitales para el desarrollo del autoconcepto y del pensamiento abstracto, por lo tanto el proceso del conocimiento de sí mismo y la concepción de la salud dependen de esta edad. ${ }^{11}$

Durante esta etapa, el niño comienza a establecer criterios en cuanto a su aspecto físico, relacionando la idea de estética a la salud; de igual manera, su concepto de bienestar y calidad de vida dependerán de las experiencias a las que el niño se enfrentó durante su vida. ${ }^{12}$

Es así como la salud bucal se involucra en el bienestar general del individuo, siendo esta un aspecto fundamental para un buen estilo de vida, ya que disponer de una buena salud bucal, no solo permite el bienestar de la persona si no también la facilidad de autorelacionarse con otros sin condiciones que lo limiten. ${ }^{13}$ Sin embargo cuando el individuo no presenta esta condición de bienestar, la caries a más de afectar la salud oral, afectaría a las condiciones de su diario vivir como el hablar, comer, dormir, entre otros. ${ }^{14}$

En los niños las alteraciones bucales causadas por caries generan un impacto negativo en su calidad vida, ocasionando procesos infecciosos, dificultad para sonreír, disminución de peso, baja autoestima y bajo desempeño escolar, es decir limitan al niño a cumplir con sus acciones habituales, comprometiendo así, el dominio de la autopercepción. ${ }^{15}$

Todo esto es evidenciado gracias a estudios que nos demuestran que esta enfermedad afecta del 60 al $90 \%$ de infantes, causando impactos tanto en su salud bucal como en su vida diaria. ${ }^{16}$

Es aquí donde nacen dos conceptos que son: calidad de vida relacionada a salud y calidad de vida relacionada a salud oral. ${ }^{17}$

En el año de 1994 la OMS define a la calidad de vida como la "percepción del individuo de su posición en la vida en el contexto de la cultura y sistema de valores en los que vive y en relación con sus objetivos, expectativas, estándares y preocupaciones". ${ }^{18}$

Podemos mencionar que el término calidad de vida en el Ecuador, se encuentra establecido en la Constitución como Sumak Kawsay o buen vivir el cual se interpreta como la relación del hombre con su entorno, la diversidad cultural y el respeto a la vida en base a todas las funciones del cuerpo humano. ${ }^{19}$

Este concepto fue experimentado en un principio en el área de salud en personas que padecían de neoplasias, describiendo de esta manera la actitud del hombre para sobrellevar a la enfermedad. ${ }^{20}$

La calidad de vida relacionada con la salud (CVRS), se define como "el impacto que una enfermedad y su tratamiento tienen sobre la percepción del paciente en su bienestar", 21 es decir, en el funcionamiento de su estado físico, social, mental y como esta influye en el diario convivir del individuo tanto con la familia así como su contacto con la sociedad. ${ }^{22}$

El concepto de calidad de vida relacionada con la salud bucal (CVRSB) se refiere al impacto de las condiciones bucales y las limitaciones que estas ocasionan tanto en la 
función normal del sistema estomatognático como en la vida diaria del hombre. ${ }^{23}$

Por esta razón la calidad de vida es una parte fundamental del diagnóstico clínico, ya que el desarrollo normal de la persona puede verse perjudicado por cualquier condición de salud bucal. ${ }^{24}$

Las entidades de Salud Pública tienen como finalidad la preservación de la calidad de vida de las personas, mediante la instauración de medidas terapéuticas y tratamientos oportunos de las enfermedades. ${ }^{25}$

Por ello la filosofía actual consiste en instaurar una cultura de la salud bucal desde el hogar que garantice una atención adecuada, persistente a partir de los primeros meses del infante, basados tanto en una nutrición como en una higiene general e higiene bucal apropiada, contando con visitas odontológicas desde el primer año del niño. ${ }^{26}$

Es por ello que este estudio tuvo como finalidad, determinar cual es la relación entre la caries y su impacto en los desempeños diarios y calidad de vida en escolares de 6 años de edad.

\section{MATERIALES Y MÉTODOS}

El estudio fue tipo descriptivo, transversal de campo y observacional. La muestra estuvo conformada por 197 estudiantes de 6 años de edad matriculados en los centros educativos pertenecientes a la parroquia San Sebastián de la ciudad de Cuenca, siendo esta muestra proporcional a la población escolar existente en la parroquia. Se consideraron como criterios de inclusión: niños que tengan 6 años de edad cumplidos o que vayan a cumplir hasta el 31 de diciembre del 2016, de ambos sexos. Los criterios de exclusión fueron: estudiantes con enfermedades sistémicas, con problemas de locomoción, con alteraciones psicológicas, con labio y paladar hendido y que no hayan aceptado el examen bucal. A su vez, el investigador recibió una calibración previa de los parámetros de diagnóstico de la OMS para caries, de tal forma que al efectuarse el examen clínico exista una interpretación similar de los mismos. Una vez establecidos estas condiciones, se procedió a dar charlas de educación para la salud bucal en las unidades educativas participantes. Para la realización del examen clínico el cual duró 6 minutos por escolar, se utilizaron todas las medidas de asepsia y antisepsia, además se contó con un lugar acondicionado para este fin, utilizando luz natural. Se inició con una entrevista directa con la población de estudio, realizando la evaluación clínica y transcribiendo toda la información obtenida en la ficha Epidemiológica de Salud Bucal de la Carrera de Odontología de la Universidad Católica de Cuenca, la misma que consta de 5 partes, sin embargo, para este estudio sólo se tomó en consideración tres partes que son datos generales del paciente, Índice de Caries CPOD/ceod y Self Report Caries. Se empezó por observar el 1er molar superior derecho (1.6), continuando con el segundo molar superior derecho deciduo (5.5) y así sucesivamente hasta llegar al 1er molar superior izquierdo (2.6), con el cual se terminó los dientes superiores. Luego se empezó con dientes los inferiores, examinando el 1er molar inferior izquierdo (3.6), luego la pieza dentaria 7.5 y así cada uno de los dientes inferiores hasta terminar en el 1er molar inferior derecho (4.6), considerando los códigos establecidos $0=$ sano, $1=$ cariado, $2=$ obturado con caries, $3=$ obturado sin caries, $4=$ perdido por caries, $5=$ perdido por otro motivo, $6=$ sellante o fisura obturada, $7=$ pilar de puente, corona, implante, $8=$ diente permanente sin erupcionar, $9=$ no registrable. Cuando se terminó de examinar cada uno de los cuadrantes se corroboró que las anotaciones hayan sido correctas. Todo esto se llevó acabo teniendo en mente la secuencia de erupción dentaria, sin tocar la boca del paciente con los dedos, examinando cada superficie del diente para determinar áreas descalcificadas, opacidades de los bordes marginales y esmalte socavado en fosas y fisuras así también se indagó al paciente la razón de la extracción dentaria. Una vez registrados los códigos en las casillas correspondientes se procedió a sumar los dientes cariados, con extracción indicada y obturados. Del resultado de estos se obtuvo el índice ceod promedio. Los resultados se reportaron como un promedio poblacional general y por sexos. Para la determinación del impacto los principales maelestares expresados por los escolares se empleó la segunda parte del cuestionario ChildIODP versión en español-Perú con la finalidad de evaluar las necesidades de la población infantil. Este abarca ocho desempeños diarios: comer, hablar, higiene bucal, dormir, sonreír, estudiar, emocional y contacto social de las cuales el niño indicó si la condición bucal "caries" afectó o no a uno de estos desempeños, mismos, que se midieron en severidad de acuerdo a la escala de Likert con valores de poco, regular y mucho, de igual manera se valoró la frecuencia en una escala de 1 a 3 , siendo 1 si la dificultad para (desempeño) se presentó una o dos veces en un mes, 2 si la dificultad para (desempeño) se presentó tres a más veces en un mes y 3 si la dificultad para (desempeño) se presentó más de tres veces en los últimos tres meses. La extensión del impacto se obtuvo determinando el número máximo de desempeños impactados por escolar en un rango de 0 (ningún desempeño diario impactado) a 8 (todos los desempeños diarios impactados). Los resultados obtenidos se reportaron como un promedio poblacional general y por sexos. Finalmente para determinar la correlación entre caries y el impacto en los desempeños diarios se empleó la prueba estadística Odds ratio. La duración de esta encuesta fue entre 8 a 10 minutos por escolar. El presente estudio contó con el permiso de la Dirección Zonal 6 de Educación del Ecuador, aprobación del Comité de Bioética de la Universidad Católica de Cuenca, consentimientos de los padres de familia y asentimientos de los escolares.

\section{RESULTADOS}

La muestra fue de 197 escolares la cual corresponde a los niños de 6 años de edad de las 11 escuelas participantes 
en este estudio. Tras clasificarla según el género se obtuvo un total del $45 \%$ de sexo femenino y un $55 \%$ de sexo masculino (véase tabla 1). El promedio total del índice ceod fue 5.25+-3, el cual según los niveles de severidad de la OMS se considera como "Alto". En relación a su distribución por género se observó que el índice ceod promedio en niños (5,39+-3) fue similar al índice ceod promedio encontrado en las niñas (5,08+-3) (véase tabla 2). En cuanto a la prevalencia del impacto de caries de acuerdo al sexo se pudo evidenciar que las niñas presentan un mayor porcentaje de impactos $(61 \%)$, presentando por lo tanto una mayor afección en comparación de los niños (52\%) (véase tabla 3). De acuerdo a los niveles de severidad se encontró que es mayor el impacto de caries $(45,5 \%)$ causado a los escolares de 6 años de edad de esta parroquia, presentándose a su vez el resto niños con menores impactos y valores significativamente disminuidos en los niveles poco con un $29,3 \%$ y regular con un 25 , $2 \%$ (véase tabla 4). De los 8 desempeños diarios el "comer" fue el más afectado con $20,4 \%$, seguido de "lavarse los dientes" con un $17,1 \%$, sin embargo los que presentaron una menor severidad de impacto fueron "hablar" con 10 , $1 \%$, "descansar" con un $11,1 \%$, "emoción" con un $11,4 \%$ "estudiar" y "sonreír" con 10,3\% y "socializar" con un 9,3\% (véase tabla 5). En cuanto a la relación entre la caries y su impacto en los desempeños diarios se determino que la mayor parte de estudiantes estuvieron enfermos de caries, lo cual demuestra que tienen 1.72 veces más probabilidades de presentar impactos en sus desempeños diarios y por ende mayor afección a en su calidad de vida que aquellos que no presentan esta enfermedad (véase tabla 6).

\section{DISCUSIÓN}

La caries dental presente en la población infantil de la Parroquia San Sebastián se enmarcó dentro de la principal causa de daños e impactos múltiples en los niños de 6 años de edad.

Es así como en este estudio se pudo comprobar la prevalencia de caries mediante la aplicación del índice ceod siendo este de 5,25 para el total de la muestra, comparándose con los valores de López ${ }^{13}$ con 3,57, Fernández et al ${ }^{14}$ con 3,27 , Medina et $\mathrm{al}^{6}$ con 2,43 y Vallejos et $\mathrm{al}^{15}$ con 2,24.

Según el género los niños son los más afectados que las niñas reportando un índice ceod promedio de 5,39 y 5,08 respectivamente, resultados similares presentaron los estudios realizados por Villalobos ${ }^{16}$ et al y Zavarce ${ }^{5}$ quienes también reportan mayor afectación con un 4,75 el primero y un 3,29 el segundo. Los valores 5,39 y 4,75 de acuerdo a los niveles de severidad establecidos por la OMS son considerados como "Alto", siendo el valor 3,29 considerado como "Moderado".

Sin embargo Torres ${ }^{17}$ en el 2010 en infantes de edad similar evidencia un alto índice ceod promedio en las niñas con un 4,69, de igual manera Gálvez ${ }^{18}$ en el 2013 presenta un promedio de 4,5 siendo considerados estos valores como “Alto". A pesar de que Martínez ${ }^{19}$ et al en el 2010 y Castillo ${ }^{20}$ en el año 2011 presentan un índice "Bajo" siguen siendo altos en comparación con el sexo masculino con valores del 1,90 y 2,15 respectivamente.

La magnitud del impacto de caries se pudo evidenciar con el $61 \%$ de niñas que presentaron dificultad para cumplir sus desempeños, siendo este valor de mayor prevalencia a los datos encontrados por Gomes ${ }^{21}(32,2 \%)$ y Kramer $^{22}$ $(16,8 \%)$.

A su vez los desempeños diarios con mayor severidad de afección fueron el comer con un 20,4\% y lavarse los dientes con un $17,1 \%$, de igual manera López ${ }^{13}$ en el 2013 indica al desempeño comer como el más prevalente con un 49,7\%, así también lo indican Abanto $^{23}$ y Gomes ${ }^{21}$ en el 2014 con un $25,7 \%$ el primero y un $13,3 \%$ el segundo. Es importante mencionar que el mínimo de impactos que manifestaron los niños de la Parroquia San Sebastián fue de 1 impacto diario y de forma máxima fueron hasta 8 impactos diarios. Estos valores obtenidos son significativos para nuestra población, pues con estas afectaciones, es imposible que un niño pueda desenvolverse en su ambiente de una manera propicia y saludable.

Tras la aplicación del cuestionario CHILD-OIDP la relación entre la caries y su impacto en los desempeños diarios se vió determinada en un promedio de 1,72 veces más de probabilidades de afección de la enfermedad de caries en la calidad de vida de los niños de esta parroquia. En reportes más recientes valores semejantes presenta la aplicación del cuestionario ECOHIS por Abanto ${ }^{24}$ (2010), Jackson ${ }^{25}$ (2011), Gomes ${ }^{21}$ (2014) y Torres et $\mathrm{al}^{26}$ (2015) en infantes de edad similar reportando un mayor impacto negativo en la calidad de vida de niños y niñas.

Se puede decir por lo tanto, que los valores de los datos presentes en este estudio tienen similitud y en algunos casos son mayores a los datos de las investigaciones científicas previas lo cual demuestra su consistencia con las mismas.

Por todo lo analizado y en vista de las grandes necesidades que se requieren en cuanto al bienestar y a la salud bucal de la población, se sugiere la realización de otros estudios epidemiológicos que no solo abarquen a nivel de parroquias si no también a nivel de cantones, provincias y de país.

\section{CONCLUSIONES}

El índice ceod fue mayor para los escolares de sexo masculino en comparación al femenino, con un promedio de 5,39 para el primero y 5,08 para el segundo.

Debido a este alto índice se pudo determinar, que la caries dental está completamente relacionada con el impacto en los desempeños diarios, influyendo a su vez, en la calidad de vida de los escolares de sexo femenino de 6 años de la Parroquia San Sebastián del cantón Cuenca. 
Tabla 1. Distribución de la muestra estudiada de acuerdo al sexo de los escolares de 6 años de la Parroquia San Sebastián Cuenca 2016.

\begin{tabular}{lcccccc}
\hline & \multicolumn{2}{c}{ Femenino } & \multicolumn{2}{c}{ Masculino } & \multicolumn{2}{c}{ TOTAL } \\
& $\mathbf{n}$ & $\%$ & $\mathbf{n}$ & $\%$ & $\mathbf{n}$ & $\%$ \\
\hline Las Pencas & 1 & 1 & 2 & 1 & 3 & 2 \\
Bell Academy & & 0 & 3 & 2 & 3 & 2 \\
Madrid & 1 & 1 & 3 & 2 & 4 & 2 \\
Ausubell School & 6 & 3 & 13 & 7 & 19 & 10 \\
Ignacio Malo Tamariz & 4 & 2 & 6 & 3 & 10 & 5 \\
Emilio Murillo & 11 & 6 & 7 & 4 & 18 & 9 \\
Ulises Chacón & 10 & 5 & 11 & 6 & 21 & 11 \\
Ángela Rodriguez & 4 & 2 & 12 & 6 & 16 & 8 \\
Reinaldo Chico A. & 11 & 6 & 13 & 7 & 24 & 12 \\
Victor Gerardo A. & 19 & 10 & 15 & 8 & 34 & 17 \\
Republica de Ecuador & 21 & 11 & 24 & 12 & 45 & 23 \\
TOTAL & 88 & 45 & 109 & 55 & 197 & 100 \\
\hline
\end{tabular}

Tabla 2. Prevalencia del índice ceod de acuerdo al sexo de los escolares de 6 años de la Parroquia San Sebastián Cuenca 2016.

\begin{tabular}{lcccc}
\hline & $\begin{array}{c}\text { Máximo de } \\
\text { ceod }\end{array}$ & $\begin{array}{c}\text { Mínimo de } \\
\text { ceod }\end{array}$ & $\begin{array}{c}\text { Promedio de } \\
\text { ceod }\end{array}$ & $\begin{array}{c}\text { Desvest de } \\
\text { ceod }\end{array}$ \\
\hline FEMENINO & 13 & 0 & 5,08 & 3,33 \\
MASCULINO & 17 & 0 & 5,39 & 3,82 \\
Total & 17 & 0 & 5,25 & 3,60 \\
\hline
\end{tabular}

Tabla 3. Prevalencia del impacto de caries de acuerdo al sexo de los escolares de 6 años de la Parroquia San Sebastián Cuenca 2016.

\begin{tabular}{lcccc}
\hline & \multicolumn{2}{c}{ Femenino } & \multicolumn{2}{c}{ Masculino } \\
& $\mathbf{n}$ & $\%$ & $\mathbf{n}$ & $\%$ \\
\hline Con Impacto & 54 & 61 & 57 & 52 \\
Sin impacto & 34 & 39 & 52 & 48 \\
TOTAL & 88 & 100 & 109 & 100 \\
\hline
\end{tabular}


Tabla 4. Niveles de severidad del impacto de caries en los escolares de 6 años de la Parroquia San Sebastián Cuenca 2016.

\begin{tabular}{lcc}
\hline $\begin{array}{c}\text { Niveles } \\
\text { De Severidad }\end{array}$ & $\mathbf{n}$ & $\%$ \\
\hline Poco & 116 & 29,3 \\
Regular & 100 & 25,2 \\
Mucho & 180 & 45,5 \\
TOTAL & 396 & 100 \\
\hline
\end{tabular}

Tabla 5. Severidad de los desempeños diarios afectados por caries en los escolares de 6 años de la Parroquia San Sebastián Cuenca 2016.

\begin{tabular}{lcc}
\hline $\begin{array}{l}\text { Desempeños } \\
\text { Diarios }\end{array}$ & $\mathbf{n}$ & $\%$ \\
\hline Comer & 81 & 20,4 \\
Hablar & 40 & 10,1 \\
Lavarse los & 67 & 17,1 \\
dientes & & \\
Descansar & 44 & 11,1 \\
Emoción & 45 & 11,4 \\
Sonreir & 41 & 10,3 \\
Estudiar & 41 & 10,3 \\
Socializar & 37 & 9,3 \\
TOTAL & 396 & 100 \\
\hline
\end{tabular}

Tabla 6. Relación entre la caries y su impacto sobre los desempeños diarios que afectan la calidad de vida de los escolares de 6 años en la Parroquia San Sebastián Cuenca 2016.

\begin{tabular}{lcccccc}
\hline & \multicolumn{2}{c}{ Enfermo } & \multicolumn{2}{c}{ Sano } & \multicolumn{2}{c}{ TOTAL } \\
& $\mathbf{n}$ & $\%$ & $\mathbf{n}$ & $\%$ & $\mathbf{n}$ & $\%$ \\
\hline Con Impacto & 98 & 50 & 13 & 6 & 111 & 56 \\
Sin impacto & 70 & 36 & 16 & 8 & 86 & 44 \\
TOTAL & 168 & 85 & 29 & 15 & 197 & 100 \\
\hline
\end{tabular}

$\mathrm{OR}=1,72$ 
Finalmente se puedo evidenciar que la severidad del impacto sobre los desempeños diarios se vió reflejada más para los desempeños "comer" y "lavarse los dientes" con un porcentaje más alto el primero a diferencia del segundo mencionado, sin embargo los demás desempeños se encuentran afectados en porcentajes menores pero de valores similares entre ellos.

Conflicto de intereses y financiamiento Los autores declaran no tener conflicto de intereses, haber cumplido con los requisitos de autoría y haber autofinanciado este artículo

\section{Referencias}

1 Catalá PM, Cortés LO. La caries dental: una enfermedad que se puede prevenir. Revista An Pedriatr Contin [Internet]. 2014 [citado Ago 27];12(3):147-151. DOCUMENTO DISPONIBLE

2 Mendes DD, Caricote LN. Prevalencia de caries dental en escolares de 6 a 12 años de edad del Municipio Antolín del Campo, Estado Nueva Esparta, Venezuela (2002-2003). Revista Latinoamericana de Ortodoncia y Ortopedia [Internet]. 2003 [citado 27 Ago 2016]; 1(1):1-20. DOCUMENTO DISPONIBLE

3 Palomer RL. Caries dental en el niño: Una enfermedad contagiosa. Revista Chilena de Pediatría [Internet]. 2006 [citado 27 Ago 2016];77(1):56-60. DOCUMENTO DISPONIBLE

4 Encala VL. Estudio Piloto Comparativo de Salud Oral relacionada a la Calidad de Vida en escolares del Cantón Cañar Ecuador 2015. Revista Odontología Activa UC Cuenca [Internet]. 2016 [citado 27 Ago 2016];1(1):1-5. DOCUMENTO DISPONIBLE

5 Zavarce ES, Izzaddin AR. Prevalencia de caries dental en menores de años de edad. Estudio en Ciudad Hospitalaria Enrique Tejera, Valencia Estado de Carabobo, Venezuela. Revista Acta Odontológica Venezolana [Internet]. 2014 [citado 27 Ago 2016]; 52(2):1-13. DOCUMENTO DISPONIBLE

6 Medina SC, Maupomé G, Pelcastre VB, Ávila BL, Vallejos SA, Casanova RA. Desigualdades socioeconómicas en salud bucal: caries dental en niños de seis a 12 años de edad. Revista de Investigación Clínica [Internet]. 2006 [citado 27 Ago 2016];58(4):296-304. DOCUMENTO DISPONIBLE

7 Rodríguez LIR, Traviesas HE, Lavandera CE, Duque HM. Factores de riesgo asociados con la caries dental en niños de círculos infantiles. Revista Cubana de Estomatología [Internet]. 2009 [citado 27 Agos 2016];46(2):1-9. DOCUMENTO DISPONIBLE

8 Núñez FL, Sanz BJ, Mejía LG. Caries dental y desarrollo infantil temprano. Estudio piloto. Revista Chilena de Pediatría [Internet]. 2015 [citado 27 Ago 2016];86(1):38-42. DOCUMENTO DISPONIBLE

9 Muñoz MP, Aguilar DF, De la Fuente HJ, Shimada BH, Acosta TL. Instrumentos validados para medir la salud bucal en los niños. Revista Siicsalud [Internet]. 2014 [citado
27 Ago 2016]; 1(1): 846-851. DOCUMENTO DISPONIBLE

0 ] Abanto JA, Bönecker M, Prócida RD. Impacto de los problemas bucales sobre la calidad de vida de niños. Revista Estomatológica Herediana [Internet]. 2010 [citado 27 Ago 2016]; 20(1):38-43. DOCUMENTO DISPONIBLE

11 Marró FM, Candiales de Castillo YM, Cabello IR, Urzúa AI, Rodríguez MG. Aspectos sobre la medición del impacto de la caries dental en la calidad de vida de las personas: Artículo de revisión. Revista Clínica de Periodoncia Implantología y Rehabilitación Oral [Internet]. 2013 [citado 27 Ago 2016];6(1):42-46. DOCUMENTO DISPONIBLE

12 Moreno RX, Vera AC, Cartes VR. Impacto de la salud bucal en la calidad de vida de escolares de 11 a 14 años, Lincantén, 2013. Revista Clínica de Periodoncia, Implantología y Rehabilitación Oral [Internet]. 2014[citado 27 Ago 2016]; 7(3): 142-148. DOCUMENTO DISPONIBLE

13 López RR, García RC. Calidad de vida y problemas bucales en preescolares de la provincia de la provincia de Huaura, Lima. Revista Estomatológica Herediana [Internet]. 2013 [citado 27 Ago 2016];23 (3): 139-147. DOCUMENTO DISPONIBLE

14 Fernández CN, Vuoto ER, Prigione C, Borjas MI, De la Rosa G, Rubio G, Giamportone V, Cabrera D, Vuoto JI. Experiencia de caries de preescolares del Gran Mendoza expresada a través de los índices ceod e índice de significación de caries. Revista de la Facultad de Odontología UNCuyo [Internet]. 2009 [citado 27 Ago 2016]; 3 (2): 3642. DOCUMENTO DISPONIBLE

15 Vallejos SA, Medina SC, Casanova RJ, Maupomé G, Casanova RA, Minaya SM. Defectos del esmalte, caries en dentición primaria, fuentes de fluoruro y su relación con caries en dientes permanentes. Revista Gaceta Sanitaria [Internet]. 2007 [citado 27 Ago 2016];21(3):227-234. DOCUMENTO DISPONIBLE

16 Villalobos RJ, Medina SC, Molina FN, Vallejo SA, Pontigo LA, Espinoza BJ. Caries dental en escolares de 6 a 12 años de edad en Sinaloa, México: experiencia, prevalencia, gravedad y necesidades de tratamiento. Revista Biomédica [Internet]. 2006 [citado 27 Ago 2016]; 26 (22): 224-231. DOCUMENTO DISPONIBLE

17 Torres LH. Estudio epidemiológico sobre caries dental y necesidades de tratamiento en escolares de 3 a 5 años de edad de Huacho Perú. Revista Salud Sexualidad y Sociedad [Internet]. 2010 [citado 27 Ago 2016]; 3 (1): 1-4. DOCUMENTO DISPONIBLE

18 Gálvez RC. Prevalencia de Caries Dental en Niños con Dentición Decidua usando índices ceo y el Sistema Internacional de Detección y Valoración de Caries (icdas II). Revista de Investigación de la Universidad Norbert Wiener [Internet]. 2013 [citado 27 Ago 2016];1(2):117-127. DOCUMENTO DISPONIBLE

19 Martínez PK, Monjarás ÁA, Patiño MN, Loyola RJ, Mandeville $\mathrm{P}$, Medina SC, Islas MA. Estudio epidemiológico sobre 
caries dental y necesidades de tratamiento en escolares de 6 a 12 años de edad de San Luis de Potosí. Revista de Investigación Clínica [Internet]. 2010 [citado 27 Ago 2016]; 62 (3): 206-213. DOCUMENTO DISPONIBLE

20 Castillo GD, García NM. Prevalencia de la caries dental en la población infantil que acuden al ambulatorio urbano "La haciendita" en el municipio de Mariara, estado de Carabobo. Revista Acta Odontológica Venezolana [Internet]. 2011 [citado 27 Ago 2016]; 49 (4): 1-7. DOCUMENTO DISPONIBLE

21 Gomes MC, Pinto ST, Costa EM, Martins CC, Granville GF. Impact of oral health conditions on the quality of life of preschool children and their families: a cross-sectional study. Revista Health Qual Life Outcomes [Internet]. 2014 [citado 27 Ago 2016]; 12(55): 1-12. DOCUMENTO DISPONIBLE

22 Kramer P, Feldens C, Ferreira S, Bervian J, Rodrigues P, Peres M. Exploring the impact of oral diseases and disorders on quality of life of preschool children. Revista Community Dentistry and Oral Epidemiology [Internet]. 2013 [citado 27 Ago 2016]; 41(4): 327-335. DOCUMENTO DISPONIBLE

23 Abanto J, Tsakos G, Paiva S, Bönecker M. Impact of dental caries and trauma on quality of life among 5- to 6-yearold children: Perceptions of parents and children. Revista Community Dentistry and Oral Epidemiology [Internet]. 2014 [citado 27 Ago 2016]; 42(5): 385-394. DOCUMENTO DISPONIBLE

24 Abanto J, Carvalho ST, Mendes F, Prócida RD. Impacto de las enfermedades orales y trastornos en la calidad de Vida relacionada con la salud oral de los niños de edad preescolar. Revista Community Dentistry and Oral Epidemiology [Internet]. 2011 [citado 27 Ago 2016]; 39 (2): 105-114. DOCUMENTO DISPONIBLE

25 Jackson S, Vann W, Kotch J, Lee J. Impacto de la mala salud oral sobre la asistencia escolar de los niños y el rendimiento. Revista American Journal of Public Health [Internet]. 2011 [citado 4 Jun 2016]; 101 (10): 45-64. DOCUMENTO DISPONIBLE

26 Torres RG, Blanco VD, Chávez SM, Apaza RS, Antezana VV. Impacto de la caries de la infancia temprana en la calidad de vida relacionada a la salud bucal en niños peruanos menores de 71 meses de edad. Revistas Odontología Sanmarquina [Internet]. 2015 [citado 27 Ago 2016];18(2):8794. DOCUMENTO DISPONIBLE

Recibido: 5 de Noviembre de 2016

Aceptado: 30 de Diciembre de 2016 